\title{
Modelling neutron star magnetic fields
}

\author{
Konstantinos $\mathbf{N}$ \\ Gourgouliatos, Rainer \\ Hollerbach and Robert F \\ Archibald describe methods \\ and outcomes of modelling \\ neutron star magnetic fields.
}

M ore than 30 years before the radiodiscovery of pulsars in 1967 (Hewish et al. 1968), Baade and Zwicky

(1934) proposed the existence of dense stars consisting primarily of neutrons as a possible outcome of stellar evolution, following a supernova explosion. Moreover, a few years prior to their discovery, it had been suggested that the otherwise dim neutron stars may host exceptionally strong magnetic fields and act as sources of electromagnetic waves (Hoyle et al. 1964). Indeed, most neutron stars emit pulses of coherent radio waves. These pulses allow very accurate timing measurements of their period and period derivatives. Isolated pulsar periods become longer with time. This is most likely caused by the torque exerted on the star by the magnetic field. In some exceptional cases spin-down may be enhanced by the emission of gravitational waves. If we assume that the magnetic field of pulsars has the simplest possible structure, that of a dipole, we can set a lower limit on its strength by equating the loss of kinetic energy to that of electromagnetic dipole emission, which gives the well-known formula of the spindown dipole magnetic field:

$$
B_{\mathrm{d}}=3.2 \times 10^{19}(P \dot{P})^{1 / 2}
$$

where $B_{\mathrm{d}}$ is the dipole magnetic field on the surface of the star measured in Gauss, the period $P$ is in seconds and the period derivative $\dot{P}$ is dimensionless. However, this estimate cannot put constraints on the presence of magnetic fields of more complex structure. In a similar way we can estimate the spin-down or characteristic age, $\tau_{\mathrm{c}}$, as the time that has elapsed for a neutron star with a given dipolar field to spin-down to its current period:

$$
\tau_{\mathrm{c}}=P /(2 \dot{P})
$$

In the earliest attempts to explain the origin of coherent radio emission (Ruderman \& Sutherland 1975, Arons \& Scharlemann 1979), it was proposed that there exist regions in the star's magnetosphere, called gaps, where the electric and magnetic fields have parallel components. Inside the gaps, the plasma is not governed by ideal magnetohydrodynamics, as is assumed for the rest of the magnetosphere. Charged particles in the gap are accelerated to high energies by the electric field, while spiraling round the magnetic field lines. As the magnetic field lines are not straight these particles emit high-energy photons as a result of curvature radiation. The photons interact with the magnetic field and produce electron-positron pairs, which annihilate and produce high-energy photons through a cascade process. The final outcome of this process is charged particles, which are responsible for the radio emission. To initiate the cascade process, the magnetic field needs to be above $10^{12} \mathrm{G}$, and the field lines' curvature radius similar to the star's radius $\left(R_{\mathrm{c}} \sim 10 \mathrm{~km}\right)$ (Gill et al. 2003). A large fraction of pulsars have much weaker magnetic fields $\left(\sim 10^{10} \mathrm{G}\right)$, but are nevertheless bright at radio wavelengths. To overcome this hurdle it was suggested that the magnetic field may also contain higher order multipoles, in addition to the large-scale dipole. These multipoles dominate near the surface of the star, but their intensity drops rapidly with distance from the pulsar. Thus, the magnetic field structure of the pulsar on large scales remains dipolar.

While the presence of higher multipoles provides a natural solution to the problem of curvature radiation, it raises the question of how these multipoles are created in the first place, and how they are sustained for millions of years. Neutron stars do not have a convection zone to host an active dynamo that will generate small-scale structure. Moreover, even if a star had been born with such small-scale features, they would have decayed within less than a million years due to the finite conductivity of the star's outer layer, its crust (Potekhin \& Chabrier 2000). Thus, even if the presence of higher multipoles is the answer to the radiation question, a mechanism is needed to create them and ensure they last long enough to make pulsars shine for millions of years.

\section{Magnetars}

Another major clue that non-dipolar field structures exist in pulsars comes from the observed behaviour of a small fraction of the pulsar population. In a tiny fraction $(-20$ of $\sim 2500)$ of the isolated pulsar population, the loss of rotational energy which explains conventional pulsar energetics is not sufficient to provide the observed energy output, nor can it explain the extreme behaviour seen from these rare sources, including orders of magnitude increases in X-ray band flux, and the emis-

$\ldots \ldots . . .$. sion of short ( $100 \mathrm{~ms})$ bursts. These rare sources are now collectively referred to as magnetars (Clark 2015, Kaspi \& Beloborodov 2017; see box "Magnetar activity").

The most extreme radiative events from magnetars are giant flares - reaching energies of $10^{44}-10^{46}$ ergs in soft gamma-rays. They are characterized by a $\sim 100 \mathrm{~ms}$ long flash, which contains approximately half of the flare's energy, followed by a minuteslong decay that is strongly modulated at the rotation period of the pulsar. Giant flares are incredibly rare; we have only seen three since the dawn of $X$-ray astronomy. The first giant flare occurred on 5 March 1979 from SGR0525-66 (Mazets et al. 1979b), the second on 27 August 1998 from SGR1900+14 (Hurley et al. 1999), and the third on 27 December 2004 from SGR 1806-20 (Palmer et al. 2005). The magnetar model (Duncan \& Thompson 1992, Thompson \& Duncan $1995,1996)$ was developed in part to explain this extreme behaviour.

The observations of bursts and flares, particularly their short rise times, imply the operation of short timescale processes. Indeed their sub-second rise timescale and incredible energy rule out any accretionbased power source. However, a strong magnetic field can provide a reservoir of stored energy that can be released rapidly with timescales as short as a fraction of a second. This rapid release implies the triggering of some instability or exceeding some critical threshold. For instance, plasma instabilities are excited when the magnetic field of the magnetosphere is twisted. Alternatively, mechanical strain is accumulated in the crust, which is 


\section{Magnetar activity}

Magnetars were initially

discovered as two classes of

objects in the early days of $\mathrm{X}$-ray

astronomy: the anomalous

$X$-ray pulsars (AXPs) and the soft gamma-ray repeaters (SGRs).

AXPs were identified as a

group of stable $X$-ray emitting

pulsars with strangely similar

periods between $\sim 5.4$ and $8.7 \mathrm{~s}$

(Mereghetti \& Stella 1995). They

were considered anomalous

in that their $\mathrm{X}$-ray luminosity

exceeded their spin-down

luminosity (Mereghetti et al. 1998).

They were observed to spin, and spin-down, much more stably than accretion-powered X-ray pulsars and, as non-detections of Doppler shifts in their spin frequencies made the limits on possible companions more and more stringent, it seemed unlikely that accretion could be the answer (Mereghetti et al. 1998).

SGRs were named for their repeated emission of soft gammaray bursts (e.g. Golenetskij et al. 1979, Mazets et al. 1979a, Golenetskii et al. 1984). Initially, these were thought to be a class of gamma-ray bursts, but their repetition and periodicity made them stand out (Mazets et al. 1979b) as distinct phenomena.

As the AXPs and SGRs were observed for longer periods of time, it was realized that the properties of these two source classes overlapped. Long-term timing showed that, like the AXPs, the SGRs possessed implied surface dipolar magnetic fields of $\sim 10^{14} \mathrm{G}$ (Kouveliotou et al. 1998). Then, in 2002, SGR-like bursts were detected from the AXP 1E 1048.1-5937 (Gavriil et al. 2002). That same year, the
AXP 1E2259+586 underwent a classic SGR-like outburst (Kaspi et al. 2003). Now, nearly all of the classic AXPs have exhibited SGR-like bursts and outbursts (Dib \& Kaspi 2014). As the properties of the two classes are now nearly indistinguishable, they are collectively referred to as magnetars. An up-to-date list of both confirmed and candidate magnetars is maintained in the McGill Magnetar Catalogue (Olausen \& Kaspi 2014; http:// www.physics.mcgill.ca/ pulsar/ magnetar/main.html). gradually deformed due to slow magnetic field evolution. Once the critical strain is reached, the crust can no longer support the stresses and releases energy rapidly. In either case, these events are ultimately driven by magnetic field evolution (Duncan \& Thompson 1992, Thompson \& Duncan 1995, 1996).

The prototypical magnetars have the highest spin-down-inferred dipolar surface magnetic fields of the neutronstar population, with fields of order $10^{14}-10^{15} \mathrm{G}$. Recently, however, magnetars with more typical inferred dipole fields have been found. For example, the discovery of magnetars with low fields, such as SGR $0418+572$ with a field of only $6 \times 10^{12} \mathrm{G}$ (Rea et al. 2010, 2013), comparable to that of an average pulsar, and Swift J1822.3-1606 with a field of $1.35 \times 10^{13} \mathrm{G}$ (Scholz et al. 2014), challenge the original magnetar definition of having a global dipolar magnetic field greater than the quantum electrodynamic field $B_{\mathrm{Q}}$.

Magnetar-like activity has also been seen in high-magnetic-field rotationpowered pulsars. The earliest indication of this came in 2006 from the young pulsar PSR J1846-0258. It emitted several magnetar-like X-ray bursts and increased its X-ray luminosity by a factor of $\sim 5$, behaviour remarkably similar to a magnetar in outburst (Gavriil et al. 2008), yet both before and after the outburst manifested itself as a typical rotation-powered pulsar. In 2016, another high-magnetic-field rotationpowered pulsar, PSRJ1119-6127, underwent a magnetar-like event, emitting several short bursts and increasing its X-ray luminosity by more than an order of magnitude (Archibald 2016).

Such magnetically powered activity from objects with lower spin-down inferred surface-dipolar magnetic fields suggests a more complex field structure than a simple dipole. Perhaps the most direct evidence of this is the variable absorption feature first observed in SGR0418+5729 (Tiengo et al. 2013). Modelling it as a proton cyclotron feature implies a local magnetic field of greater than $2 \times 10^{14} \mathrm{G}$, much greater than the implied dipolar field of $6 \times 10^{12} \mathrm{G}$ (figure 1). A similar feature has been claimed in another low-B magnetar, Swift J1822.3-1606 (Rodríguez Castillo et al. 2016).

These ultra-strong fields also change the persistent emission properties of the star. Compared to rotation-powered pulsars of similar ages, the magnetars tend to be hot, having temperatures of $\sim 0.5-1 \mathrm{keV}$ (e.g. Olausen \& Kaspi 2014). The soft X-ray component of magnetar spectra can be modelled as thermal emission from the surface of the star. According to these models, the emitting region is a small, kilometre-sized spot, whose temperature is approximately an order of magnitude higher than the surface temperature of the rest of the star (Viganò et al. 2013). Given the high thermal conductivity of the crust, one would expect the surface temperature to be approximately uniform. Indeed, this would be the case for a non-magnetized neutron star. A strong magnetic field on the contrary breaks the spherical symmetry of the system, but also provides the essential source of power. This could take place either via the acceleration of particles through arcades of electric currents, the so-called j-bundles, or via ohmic heating released by the magnetic field decay in the crust. In the j-bundle case, charged particles bombard and heat the crust. If the X-ray emission comes from ohmic heating, the field decays because the conductivity of the crust is finite. While a dipolar magnetic field could lead to the necessary asymmetry, it cannot provide sufficient energy to power the weaker field magnetars whose dipole strength is below $10^{14} \mathrm{G}$. The electric current is proportional to the curl of the magnetic field, so a field with a given intensity but with small-scale structure will be supported by stronger electric currents. This configuration will provide a higher dissipation rate and higher power, albeit

\section{....... for a shorter time. Because} for a shorter time. Because
magnetars are young sources though, this shorter time is not an issue. Finally, considering the observations of proton cyclotron features in some magnetars, smaller scale magnetic fields are not only present but their strength can be higher than the dipole component (Tiengo et al. 2013).

Thus, both magnetars and to some extent normal pulsars have a magnetic field structure that is probably more complicated than a dipole. Moreover, explosive events in magnetars hint towards magnetic field evolution.

\section{Hall effect}

Despite their name, neutron stars do not consist only of neutrons. Their crust is about $1 \mathrm{~km}$ thick and comprises a highly conducting ion lattice (Lattimer \& Prakash 2001). In this part of the star, the magnetic field evolves primarily due to the classical Hall effect. The textbook example of the Hall effect is that of a ribbon-shaped conductor running perpendicular to an externally imposed magnetic field. As a current flows along the conductor, electrons experience a Lorentz force that deflects them towards one side of the ribbon, but cannot displace the ions which are fixed in the lattice. Because of this, the side where the electrons are concentrated becomes negatively charged and the other side positively charged. Thus, a Hall voltage appears between the edges of the ribbon. 

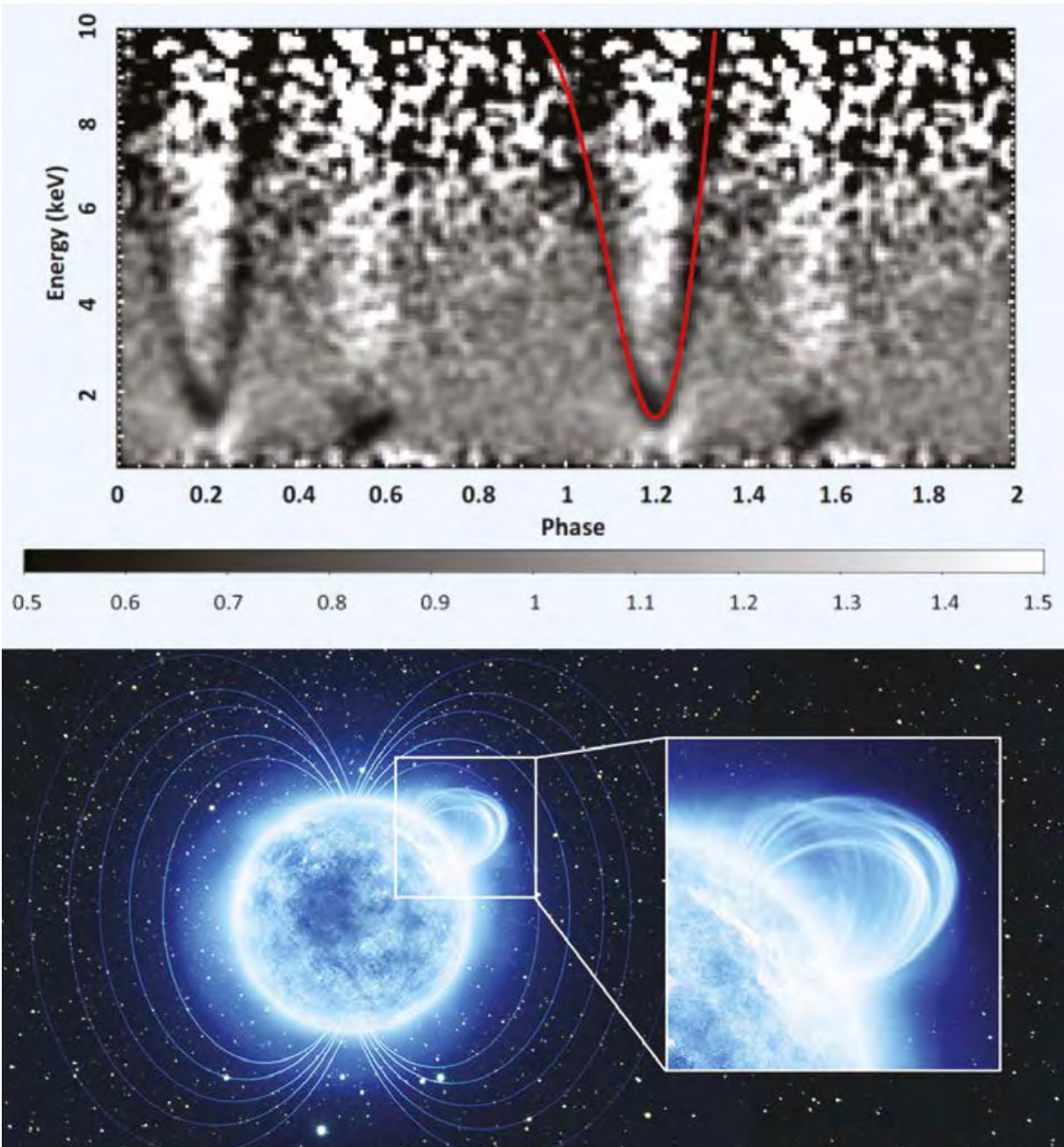

1 (Top) Phase and energy resolved light-curve. As the neutron star rotates, the intensity of X-rays drops in the part between 1 and $5 \mathrm{keV}$, appearing darker in the plot; the absorption feature in SGR0418+5729 is highlighted by a red line (Tiengo et al. 2013). (Bottom) Artist's impression of the magnetic field arcade responsible for the absorption feature. (ESA)

In the neutron star context, the operation of the Hall effect on the overall field evolution is more complicated. There, the magnetic field and electric current are not externally imposed, but are related to each other through Ampère's law: essentially the magnetic field corresponds to a unique electric current distribution. Moreover, as the currents change, the magnetic field evolves, closing this nonlinear feedback loop. Expressing this in mathematical form, the electric current $J$ is related to the electron number density $n_{\mathrm{e}}$ and velocity $v$

$$
J=-e n_{\mathrm{e}} \boldsymbol{v}
$$

where $e$ is the elementary charge. Then by Ampère's law

$$
J=c /(4 \pi) \nabla \times B
$$

where $\boldsymbol{B}$ is the magnetic field and $c$ the speed of light. From Ohm's law we can express the electric field in terms of the magnetic field and the electric current:

$$
E=-(v / c) \times B+J / \sigma
$$

Finally, substituting into Faraday's law, we obtain the Hall induction equation (Goldreich \& Reisenegger 1992)

$$
\frac{\partial \boldsymbol{B}}{\partial t}=-\frac{c}{4 \pi} \nabla \times\left[\frac{\nabla \times \boldsymbol{B}}{e n_{\mathrm{e}}} \times \boldsymbol{B}+\frac{c}{\sigma} \nabla \times \boldsymbol{B}\right]
$$

The first term inside the bracket describes the evolution of the field due to the Hall effect, and the second one the ohmic dissipation. The Hall term is nonlinear, and can be thought of as the advection of the magnetic flux by the electron fluid. It is also conservative, that is, it does not change the total magnetic energy. The ohmic term on the contrary is linear, and leads to the dissipation of magnetic energy.

From the Hall induction equation we can define a timescale on which the Hall effect acts, $t_{\mathrm{H}}=4 \pi e n_{\mathrm{e}} L^{2} /(c B) \approx L_{\mathrm{km}}^{2} n_{\mathrm{e}, 36} B_{14}^{-1} 10^{6} \mathrm{yr}$, where $B_{14}$ is the magnetic field divided by $10^{14} \mathrm{G}$, which is a typical dipole strength for a magnetar, $L_{\mathrm{km}}$ is the characteristic lengthscale of the field in kilometres, $n_{\mathrm{e}, 36}$ is the electron number density divided by its value at the base of the crust, $10^{36} \mathrm{~cm}^{-3}$. This timescale is reduced for stronger fields and for locations closer to the surface of the star where the electron number density drops to lower values.

The nonlinear nature of the Hall term suggests that the evolution of the magnetic field will be highly non-trivial. Three basic directions have been explored in the literature: turbulence, instabilities and secular evolution.
Turbulence, instabilities, secular evolution It is interesting to compare the Hall induction equation (6) with the vorticity equation of ordinary fluid dynamics

$$
\partial \boldsymbol{\omega} / \partial t=\nabla \times(\boldsymbol{u} \times \boldsymbol{\omega})+R_{\mathrm{e}}^{-1} \nabla^{2} \boldsymbol{\omega}
$$

where $u$ is the fluid velocity, $\omega=\nabla \times u$ is the vorticity, and $R_{\mathrm{e}}$ is the Reynolds number.

If the electron number density $n_{\mathrm{e}}$ and the conductivity $\sigma$ are taken to be constant, and $\boldsymbol{b}$ is the nondimensionalized magnetic field, then (6) becomes

$$
\partial \boldsymbol{b} / \partial t=-\nabla \times(j \times \boldsymbol{b})+R_{\mathrm{B}}^{-1} \nabla^{2} \boldsymbol{b}
$$

where $j=\nabla \times \boldsymbol{b}$ and the Hall parameter $R_{\mathrm{B}}=\sigma B / e n_{\mathrm{e}} \mathrm{C}$, with $B$ a typical field strength. Inserting $B=10^{14} \mathrm{G}$, and suitable crustal values for $n_{\mathrm{e}}$ and $\sigma$, yields $R_{\mathrm{B}} \sim 10^{2}-10^{3}$. In fact both $n_{\mathrm{e}}$ and $\sigma$ vary substantially throughout the depth of the crust; we will see below that variations in $n_{\mathrm{e}}$ in particular have dynamical consequences beyond just altering the local value of $R_{\mathrm{B}}$.

Comparing equations (8) and (7) shows that the two nonlinear terms are superficially similar, with the main difference being that in (8) $j$ is the curl of $\boldsymbol{b}$, whereas in (7) $u$ is the "inverse-curl" of $\boldsymbol{\omega}$. (The change in sign between the two nonlinear terms is not important; the minus sign in (8) could be removed simply by defin$\operatorname{ing} \tilde{\boldsymbol{b}}=-\boldsymbol{b}$ and $\tilde{\boldsymbol{j}}=-j$.) Based in part on this similarity between these two evolution equations, Goldreich and Reisenegger (1992) suggested that (8) and thus also (6) would initiate a turbulent cascade to small length scales, thereby providing a natural explanation for the existence of complex structures in the magnetic field.

However, the difference between "curl" and "inverse-curl" also yields some fundamental differences between these two equations. In the vorticity equation (7), the dissipative term contains more derivatives than the nonlinear term, so on sufficiently short length scales diffusion always dominates, resulting in the familiar Kolmogorov dissipation scale. In contrast, in the magnetic induction equation (8), the Hall and ohmic terms both contain two derivatives, so conceivably the Hall term could dominate on arbitrarily short length scales. A dissipative cutoff would exist only if the cascade is local in wavenumber space, since a local Hall parameter $R_{\mathrm{B}}$ could then be defined based on the field strength at that length scale only, and for sufficiently short length scales this local $R_{\mathrm{B}}$ would eventually become less than unity. This would therefore introduce a natural length scale where diffusion dominates, even though the original definition of $R_{\mathrm{B}}$ does not involve any length scales directly (Hollerbach \& Rüdiger 2002, 2004).

Periodic-box simulations in both two and three spatial dimensions (Wareing \& Hollerbach 2009, 2010) show no signs of any such dissipative cutoffs, strongly suggesting that the coupling is not local in 
wavenumber space, and that the Hall effect is instead capable of efficiently coupling widely disparate length scales. This is in stark contrast to familiar hydrodynamic turbulence, where Galilean invariance means that the smallest eddies are simply advected along by the flow on the largest scales, with little interaction otherwise. Despite these differences in local versus non-local coupling, the numerically computed Hall spectra do look remarkably like turbulent spectra, and are indeed quite close to the $k^{-2}$ spectra originally predicted by Goldreich \& Reisenegger (1992).

Another difference from ordinary turbulence that was revealed by these periodicbox simulations is that the solutions become essentially frozen in place, in the sense that the spectra appear turbulent, but the actual structures in real space hardly move, even retaining certain aspects of the (random) initial conditions. In contrast, in hydrodynamic turbulence no individual eddy maintains its position for long, or even continues to exist. In a periodic-box geometry there is of course no preferred position, so whatever the solutions freeze into is dictated simply by whatever random initial conditions were chosen.

In contrast, in the spherical shell geometry more relevant to astrophysics, any large-scale dipole component immediately distinguishes certain positions as magnetic poles versus equator. In this configuration it was found that a range of different initial conditions all evolved towards what was called a Hall attractor (Gourgouliatos \& Cumming 2014), and that once in this state, the freezing-in effect largely maintained the structure, even though ohmic decay alone would otherwise have caused different multipole components to decay at different rates.

Another important question concerns the possibility that such a quasi-equilibrium solution could become unstable, and what might happen in such a situation (Rheinhardt \& Geppert 2002). Of particular interest is the speed at which such instabilities could develop, the total amount of energy liberated, and whether this could be related to observations of magnetar bursts. Numerical calculations in local box geometry (Gourgouliatos et al. 2015, Gourgouliatos \& Hollerbach 2016) indicate that localized spots with fields up to $10^{15} \mathrm{G}$ can develop, and can release up to $10^{42} \mathrm{erg}$ via ohmic heating within a few days, consistent with observed outbursts.

Returning to the full equation (6), the electron number density $n_{\mathrm{e}}$ varies significantly throughout the depth of the crust, from $10^{36} \mathrm{~cm}^{-3}$ at the base of the crust to $10^{33} \mathrm{~cm}^{-3}$ at the surface. This has significant implications not just for local instabilities (Gourgouliatos et al. 2015, Wood et al. 2014),
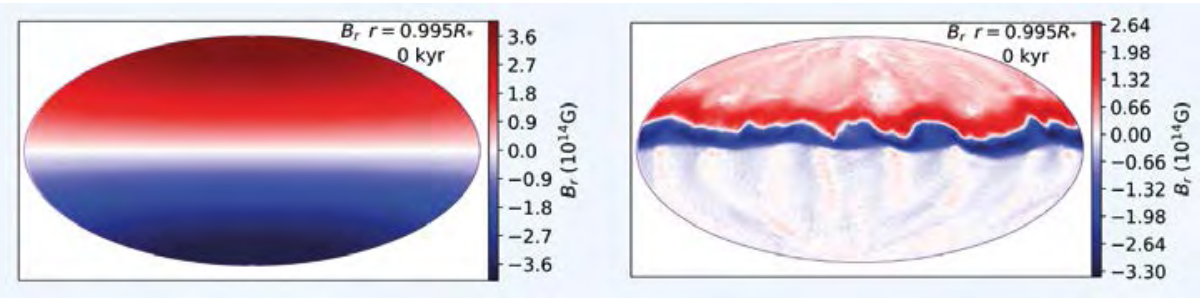

2 Radial component of the magnetic field on the surface of a neutron star at the beginning of the numerical simulation (left) and at $550 \mathrm{kyr}$ (right) of a neutron star's life. In this simulation the magnetic field's poloidal and toroidal components contain equal amounts of energy. The magnetic field remains predominantly axisymmetric with some non-axisymmetric imprints seen at the equatorial band.

but also for the large-scale dynamics. The Hall term in (6) is such that the longitudinal field component $\boldsymbol{B}_{\phi}$ interacts with the radial gradient of $n_{\mathrm{e}}$ to produce a large-scale drift of the field in the latitudinal direction (Vainshtein et al. 2000). The direction of this drift is determined by the sign of $\boldsymbol{B}_{\phi}$, with positive/negative $\boldsymbol{B}_{\phi}$ yielding motion in the north/south directions. This leads to various interesting possibilities: if $\boldsymbol{B}_{\phi}$ has the same sign in both hemispheres, everything eventually gets swept towards one of the poles. Reversing the sign of $\boldsymbol{B}_{\phi}$ would sweep everything towards the other pole, consistent with the fact that reversing the sign of such a $\boldsymbol{B}_{\phi}$ field is equivalent to simply turning the whole star upside down.

In contrast, suppose the equatorial symmetry of $\boldsymbol{B}_{\phi}$ is such that it has the opposite sign in the two hemispheres. In this case stars with fields in the upper and lower hemispheres represented by " \pm " and “ $\mp$ " are fundamentally different; turning either configuration upside down yields itself again, rather than the other one. Correspondingly, the dynamics are also different, with \pm sweeping field lines away from the equator toward the poles, whereas $\mp$ concentrates the field in a ring around the equator (Hollerbach \& Rüdiger 2004).

Furthermore, it is not clear which of these three possibilities, \pm , $\mp$, or a single sign in both hemispheres, is most relevant to real neutron stars. If we assume that the largescale poloidal field is predominantly dipolar, and that the azimuthal field is largely just that produced by the Hall effect itself, then $\boldsymbol{B}_{\phi}$ turns out to have the $\mp$ symmetry, yielding an equatorial ring. If, however, there is a significant pre-existing $\boldsymbol{B}_{\phi}$ component, such as might result from differential rotation in the proto-neutron star phase, then \pm could be just as likely. (Having the same sign in both hemispheres is probably least likely, because it would typically be associated with a quadrupolar symmetry for the large-scale poloidal field.)

In summary, the magnetic field in the crusts of neutron stars evolves as a result of the Hall effect. Despite the Hall effect conserving magnetic energy, it can accelerate the dissipation of the field by generating smaller-scale structures and facilitating ohmic decay. Quite remarkably, its operation becomes less important as the evolution progresses, an effect that becomes evident in the so-called Hall attractor state where the Hall effect gets saturated. There, the Hall parameter $R_{\mathrm{B}}$ is still much greater than unity, but the system freezes into a slowly decaying quasi-equilibrium.

\section{Spots and multipoles}

Let us now consider the full three-dimensional evolution of the magnetic field in the crust of a neutron star. In these situations the critical parameter is the energy contained in the initial poloidal and toroidal components. If the initial toroidal field is ........ weak, the overall structure remains predominantly axisymmetric, as in figure 2 , and evolves in ways similar to simulations with imposed axisymmetry (Wood \& Hol-

lerbach 2015). This is mainly because of the overall azimuthal current flowing within the crust. This current sweeps out any nonaxisymmetric features that may develop, and the field maintains the symmetry of the initial conditions.

A more interesting case appears when a strong tangential field is included in the initial conditions, in the form of a predominantly axisymmetric azimuthal field (Gourgouliatos et al. 2016, Gourgouliatos \& Hollerbach 2018). If axisymmetry is enforced in this scenario, the field will concentrate towards the poles (Geppert \& Viganò 2014), depending on its sign, as discussed above. However, this picture becomes more complex when three-dimensional evolution is allowed. In this setup, while the field moves towards the poles, it also develops the so-called Hall densityshearing instability (Wood et al. 2014). In the Cartesian geometry picture of this instability it is found that it operates when a unidirectional magnetic field of varying strength lies within a conductor with stratified electron number density. The instability is favoured if the electron number density gradient is perpendicular to the direction of the magnetic field and parallel to the 

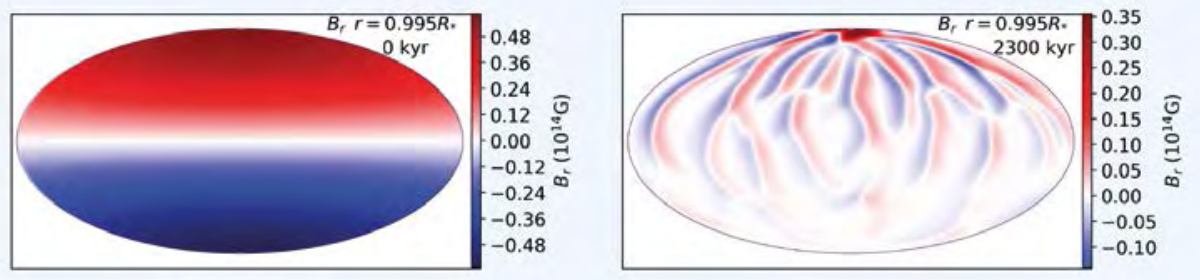

3 Radial component of the magnetic field on the surface of a neutron star at the beginning of the numerical simulation (left) and at $2300 \mathrm{kyr}$ (right) of a neutron star's life as in figure 2. In this simulation the initial toroidal magnetic field contains $99 \%$ of the energy. The magnetic field is advected to the northern hemisphere, as expected from axisymmetric theory, while non-axisymmetric structure appears as a result of the instability in the form of meridional bands.

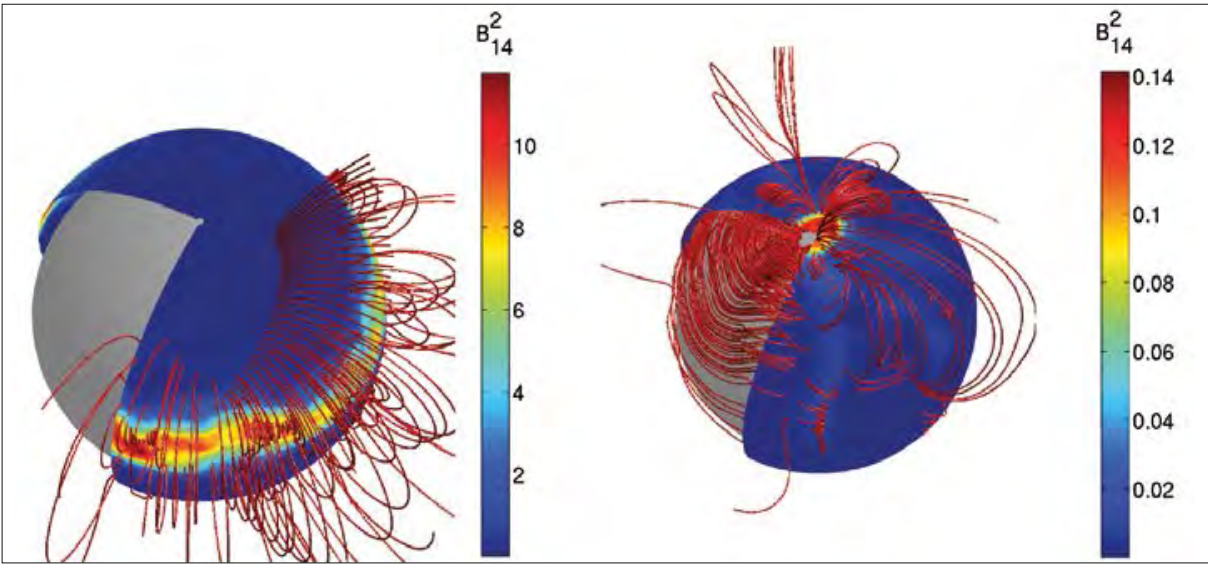

4 The magnetic field structure at the end of simulations where $50 \%$ (left) and $99 \%$ (right) of the energy is in the toroidal component of the magnetic field. In the weaker toroidal field configuration the structure of the field is predominantly axisymmetric. On the contrary, in the stronger toroidal field simulation a spot forms around the pole and a larger fraction of the magnetic field is non-axisymmetric.

direction along which the field changes. The instability generates helical waves whose wavenumber is parallel to the direction of the field. The configuration that leads to the most rapid growth of the instability corresponds to a field that varies at a similar scale as the electron number density. Such conditions are realized when the magnetic field contains a strong azimuthal component. Indeed, the direction of such a field is perpendicular to the radius of the star, while the gradient of the electron number density is parallel to the radius. Thus, at the same time as the field migrates towards the poles, it also generates non-axisymmetric features. What determines the end point of this evolution is the ratio of initial poloidal versus toroidal field. Having $50 \%$ of the energy in the toroidal field would only lead to some minor displacement from the equator, with a stronger field in a zone at mid and lower latitudes. Magnetic fields containing more energy in the toroidal component $(90 \%$ and $99 \%$ ) will push the field closer to the poles and generate more non-axisymmetric structure. In the initial transitional phase, the density-shearing instability becomes activated, generating zones of incoming and outgoing magnetic field. If the initial toroidal field contains $90 \%$ of the total energy, the field will generate a region around the poles where the strength of the field is about an order of magnitude higher than the dipole field. Initial states with even more energy in the toroidal field ( $99 \%$ ) lead to the formation of a magnetic spot where the field strength is about two orders of magnitude higher than that of the dipole field, as in figure 3. What is interesting for the formation of spot-like structures is that they mostly depend on the ratio between the poloidal and toroidal energy rather than the overall field strength. For instance, a configuration where $99 \%$ of the energy is in the toroidal field generates magnetic spots more easily than initial conditions with $90 \%$ of the energy in the toroidal, even if the latter has a field which is overall an order of magnitude stronger. Given the nonlinear nature of the Hall evolution, changing the strength of the field does not lead to a simple scaling on the strength and timescales of evolution, but to different evolution channels.

For instance, increasing the overall strength of the magnetic field in the setup where $90 \%$ of the energy is in the toroidal field leads to a more asymmetric field near the pole compared with the more uniform field found when the field is weaker by a factor of four. Since the formation of spots depends primarily on the fraction of the energy in the toroidal field, they can appear in neutron stars whose dipole field is well below the magnetar range and otherwise observed as normal pulsars, see figure 4 .

The resulting structure has a unique decomposition to multipoles comprising the power spectrum of neutron star magnetic field. The size of the magnetic spots is evident in the multipoles whose order corresponds to the appropriate wavenumber. In addition to the higher multipole components, the dipole field is still present. Note, however, that the location of the dipole axis does not coincide with that of the magnetic spot generated. Because this mechanism generates nonaxisymmetric field, the dipole axis also drifts with respect to its initial location. This drift is a slow erratic motion and becomes higher when the dipole direction does not coincide with the axis of symmetry of the initial toroidal field. In this case the magnetic dipole axis may drift by more than $45^{\circ}$ within a million years.

In accordance with box and axially symmetric spherical shell simulations where the Hall evolution saturates, a similar effect appears here. Indeed, the magnetic field changes rapidly at first, dissipating a large fraction of the energy and generating smaller scale structure. Later though, the features that have been created persist in time with minimal changes in appearance except for the overall ohmic decay.

\section{The neutron star zoo}

The diversity of the neutron star population remains a great puzzle. The grand unification of neutron stars, namely to investigate whether the life of a neutron star is determined by its properties at birth, primarily its magnetic field, is one of the main challenges in the physics of neutron stars.

The ensemble of the pulsar population can be visualized in the period-period derivative diagram, usually abbreviated as the $P-\dot{P}$ diagram, shown in figure 5 . The central part of this diagram is occupied by the rotationally powered radio pulsars, which are most common. The top left region contains younger pulsars while the top right part contains magnetars. Several magnetars can be associated with supernova remnants (SNR), confirming their young ages. Another puzzling class of neutron stars that have been found near the centre of SNRs are the central compact objects (CCOs). These neutron stars have weak dipole inferred magnetic fields $\left(B<10^{12} \mathrm{G}\right)$, yet they are hot enough to be visible in $X$-rays. The bottom right part appears uninhabited beyond the socalled death line, with very few neutron stars appearing in this region, and most of them seen through $\mathrm{X}$-rays rather than radio emission, the so-called $X$-ray dim isolated neutron stars (XDINS). Finally, in the bottom left, millisecond pulsars can be found. Millisecond pulsars are most likely to be recycled pulsars: older neutron stars which are members of binary systems that 


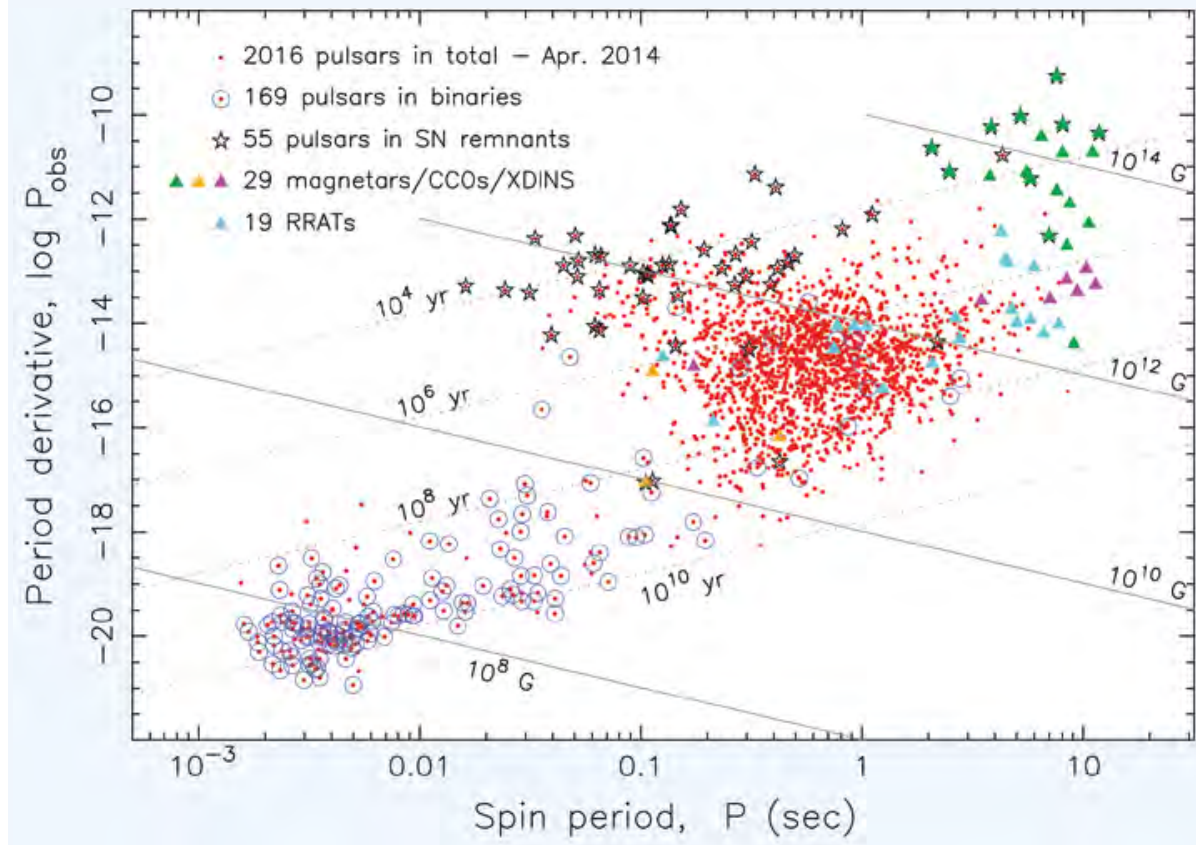

5 The $P-\dot{P}$ diagram containing the whole pulsar population. Diagonal solid lines indicate constant spindown dipole magnetic field and dotted lines constant characteristic age. Various colours are used for different types of neutron stars. (Figure from Tauris et al. 2015)

have been spun-up via accretion from their companions. A neutron star whose dipole field, radius and moment of inertia do not change in time will follow trajectories along diagonal lines of constant $P-\dot{P}$. If the magnetic field decays, the trajectories will no longer be straight lines. Depending on how rapidly the field decays compared with its characteristic age, these trajectories bend downwards. Population synthesis studies have approached this question with some of them favouring magnetic field evolution (Popov et al. 2010).

While unifying the neutron star population in its entirety is yet to be achieved, several important conclusions can be drawn by considering the Hall evolution of the magnetic field. The decay of the magnetic field caused by the combination of Hall drift and ohmic dissipation can relate the magnetar to the XDINS population. In this picture, magnetars are younger neutron stars whose magnetic field undergoes a drastic reconfiguration. As a result, their magnetic field decays rapidly and heats them, while strong stresses are also building up in the crust (Pons \& Perna 2011). These stresses are responsible for the most explosive events, which happen early in magnetars' lives. Once the reconfiguration of the magnetic field is complete the Hall effect saturates. At this stage the magnetic field relaxes to a Hall attractor state; then, while the magnetic field is still rather strong, it decays at the slower ohmic rate and its structure remains frozen in the crust. Consequently it releases less heat and explosive events are rare. Low magnetic field magnetars can be accommodated in this picture as neutron stars with strong

toroidal fields and relatively weaker dipole fields. Since the dipole field is what we can estimate through spin-down measurement, this could give the false impression that the overall magnetic field is weak, whereas in reality the non-dipolar components can be in the magnetar range.

Finally, the Hall effect may have implications for the normal pulsar population. Neutron stars with dipole components in the range of $10^{10}-10^{12} \mathrm{G}$ may still have a toroidal field whose strength is $\sim 10^{13} \mathrm{G}$. While this toroidal field will not have an obvious observational impact - it is too weak to deform the crust and lead to magnetar behaviour - it can still lead to the formation of localized stronger magnetic fields in the form of spots and multipoles. These magnetic spots will serve as sites of initial particle acceleration which eventually lead to the cascades needed for the creation of coherent radio emission.

While the impact of the Hall effect on the crustal magnetic field is becoming clearer, further steps are needed to achieve a global understanding of magnetic field structure and evolution in neutron stars. The physics of the inner part of the crust and the core need to be assessed, especially with respect to ambipolar diffusion and superconductivity. Moreover, the crust itself may not be static but evolves slowly in response to the changes of the magnetic field and its rotation frequency; could these changes affect the evolution of the field itself? Finally, the magnetosphere comprises a complicated electric circuit with twisted magnetic fields, electric currents and charged regions; an important question here is how exactly the magnetosphere is coupled to the neutron star and how it reacts to internal magnetic field evolution. Answering these questions will open new paths and brings us closer to understanding these wonderful objects. $\bullet$

88101103

Rodríguez-Castillo GA et al. 2016 Mon. Not. R Astron. Soc. 4564145

Ruderman MA \& Sutherland PG 1975 Astrophys. J. 19651

Scholz P et al. 2014 Astrophys. J. 78662

Tauris TM et al. 2015 Advancing Astrophysics with the Square Kilometre Array (AASKA14)039

Thompson C \& Duncan RC 1995 Mon. Not. R. Astron. Soc. 275255

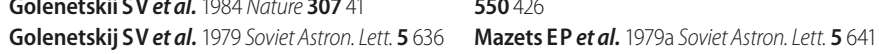
Gourgouliatos KN \& Cumming A 2014 Phys. Rev. Mazets EP et al. 1979b Nature 282587

Letts 88101103

Gourgouliatos KN \& Hollerbach R 2016 Mon

Not. R. Astron. Soc. 4633381

Gourgouliatos KN \& Hollerbach R 2018 Astrophys. J. 85221

Gourgouliatos KN et al. 2015 Mon. Not. R. Astron. Soc $453\llcorner 93$

Gourgouliatos KN et al. 2016 Proc. Nat. Acad. Sci. 1133944

Hewish A et al. 1968 Nature 217709 Hollerbach R \& Rüdiger G 2002 Mon. Not. R. Astron. Soc. 337216

Hollerbach R \& Rüdiger G 2004 Mon. Not. R. Astron. Soc. 3471273
Mereghetti S \& Stella L 1995 Astrophys. J. 442 L17 Mereghetti S et al. 1998 Mon. Not. R. Astron. Soc. 296689

Olausen SA \& Kaspi V M 2014 Astrophys. J. Supp. 2126

Palmer DM et al. 2005 Nature 4341107 Pons JA \& Perna R 2011 Astrophys. J. 741123 Popov SB et al. 2010 Mon. Not. R. Astron. Soc. 4012675

Potekhin A Y \& Chabrier G 2000 Phys. Rev. E 62 8554

Rea N et al. 2010 Science 330944

Rea N et al. 2013 Astrophys. J. 77065

Rheinhardt M \& Geppert U 2002 Phys. Rev. Letts
Thompson C \& Duncan RC 1996 Astrophys. J.

473322

Tiengo A et al. 2013 Nature 500312

Vainshtein Sl et al. 2000 Phys. Rev. E 614422 Viganò D et al. 2013 Mon. Not. R. Astron. SoC. 434123

Wareing C J \& Hollerbach R 2009 Phys. Plasmas 16042307

Wareing C J \& Hollerbach R 2010 J. Plasma Phys. 76117

Wood TS \& Hollerbach R 2015 Phys. Rev. Letts 114191101

Wood TS et al. 2014 Phys. Plasmas 21052110 